\title{
PUMA mediates ER stress-induced apoptosis in portal hypertensive gastropathy
}

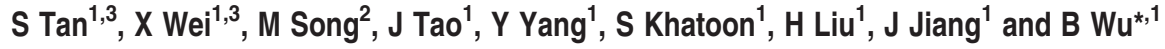

Mucosal apoptosis has been demonstrated to be an essential pathological feature in portal hypertensive gastropathy (PHG). p53-upregulated modulator of apoptosis (PUMA) was identified as a BH3-only Bcl-2 family protein that has an essential role in apoptosis induced by a variety of stimuli, including endoplasmic reticulum (ER) stress. However, whether PUMA is involved in mucosal apoptosis in PHG remains unclear, and whether PUMA induces PHG by mediating ER stress remains unknown. The aim of the study is to investigate whether PUMA is involved in PHG by mediating ER stress apoptotic signaling. To identify whether PUMA is involved in PHG by mediating ER stress, gastric mucosal injury and apoptosis were studied in both PHG patients and PHG animal models using PUMA knockout (PUMA-KO) and PUMA wild-type (PUMA-WT) mice. The induction of PUMA expression and ER stress signaling were investigated, and the mechanisms of PUMA-mediated apoptosis were analyzed. GES-1 and SGC7901 cell lines were used to further identify whether PUMA-mediated apoptosis was induced by ER stress in vitro. Epithelial apoptosis and PUMA were markedly induced in the gastric mucosa of PHG patients and mouse PHG models. ER stress had a potent role in the induction of PUMA and apoptosis in PHG models, and the apoptosis was obviously attenuated in PUMA-KO mice. Although the targeted deletion of PUMA did not affect ER stress, mitochondrial apoptotic signaling was downregulated in mice. Meanwhile, PUMA knockdown significantly ameliorated ER stress-induced mitochondria-dependent apoptosis in vitro. These results indicate that PUMA mediates ER stress-induced mucosal epithelial apoptosis through the mitochondrial apoptotic pathway in PHG, and that PUMA is a potentially therapeutic target for PHG.

Cell Death and Disease (2014) 5, e1128; doi:10.1038/cddis.2014.95; published online 13 March 2014

Subject Category: Experimental Medicine

Portal hypertensive gastropathy (PHG) occurs as a serious complication of liver cirrhosis and is a potential cause of gastric hemorrhage in patients afflicted with cirrhosis. ${ }^{1-3}$ Numerous studies have determined that in PHG, the gastric mucosa has an increased susceptibility to injury caused by alcohol, non-steroidal anti-inflammatory drugs (NSAIDs) and other noxious factors. ${ }^{4,5}$ Furthermore, the alterations in critical factors accompanying portal hypertension (PHT) such as prostaglandins, tumor necrosis factor- $\alpha$ (TNF- $\alpha$ ), nitric oxide (NO), Endothelin-1 and transforming growth factor- $\alpha$ (TGF- $\alpha$ ) have also been described to participate in the formation of $\mathrm{PHG}^{6-8}$ However, the pathogenesis of this disorder has not been fully explored.

Our previous data demonstrated that gastric mucosal apoptosis was involved in PHG, and many other studies also showed that cell death has a pivotal role in the development of PHG. ${ }^{7,8}$ Apoptosis has been implicated in tissue damage under a number of pathological conditions. ${ }^{9,10}$ The $\mathrm{Bcl}-2$ family proteins include evolutionarily conserved key modulators that mediate apoptosis through the mitochondrial pathway. p53-upregulated modulator of apoptosis (PUMA), a BH3-only $\mathrm{Bcl}-2$ family protein, is one of the most potent mediators of p53-dependent and p53-independent apoptosis induced by various stimuli. ${ }^{11,12}$ PUMA transduces death signals primarily to the mitochondrial membrane, where it spurs the translocation of Bax and Bak, the release of cytochrome $c$ then leads to caspase activation, and ultimately the cells die. ${ }^{13}$ PUMA knockout (PUMA-KO) in human colon cancer cells or in mice leads to marked attenuation of mitochondrial apoptosis induced by multiple stimuli such as DNA damage, gamma irradiation, kinase inhibition, oncogene activation and endoplasmic reticulum (ER) stress. ${ }^{14}$

The mitochondrial apoptotic pathways have also been widely shown to be linked to ER stress in recent studies. ${ }^{15,16}$ ER stress, also known as the unfolded protein response (UPR), is a phenomenon that occurs when excessive protein

\footnotetext{
1Department of Gastroenterology, The Third Affiliated Hospital of Sun Yat-Sen University, Guangzhou, China and ${ }^{2}$ Department of Pharmacy, The Third Affiliated Hospital of Sun Yat-Sen University, Guangzhou, China

*Corresponding author: B Wu, Department of Gastroenterology, The Third Affiliated Hospital of Sun Yat-Sen University, 600 Tianhe Road, Guangzhou 510630, China. Tel: +86 20 85252801; Fax: +86 20 85253336; E-mail: binwu001@ hotmail.com

${ }^{3}$ These authors contributed equally to this work.

Keywords: PHG; PUMA; ER stress; mitochondria; apoptosis

Abbreviations: PHG, portal hypertensive gastropathy; $\mathrm{PHT}$, portal hypertension; $\mathrm{SO}$, sham-operated; $\mathrm{PVL}$, partial portal vein ligation; $\mathrm{CCl}_{4}$, carbon tetrachloride; PUMA, p53-upregulated modulator of apoptosis; ER, endoplasmic reticulum; UPR, unfolded protein response; GRP78, glucose regulated protein 78; sXBP1, spliced X-box protein-1; elF2 $\alpha$, eukaryotic initiation factor 2 alpha; p-elF2 $\alpha$, phosphorylated eukaryotic initiation factor 2 alpha; ERp72, endoplasmic reticulum protein 72; IRE-1 $\alpha$, inositol requiring- $1 \alpha$; PERK, protein kinase RNA-like endoplasmic reticulum kinase; ATF- $6 \alpha$, activating transcription factor- $6 \alpha$; NSAIDs, non-steroidal antiinflammatory drugs; TNF- $\alpha$, tumor necrosis factor- $\alpha$; NO, nitric oxide; TUNEL, terminal deoxynucleotidyl transferase-mediated deoxyuridine triphosphate nick end labeling; siRNA, small interfering RNA; IHC, immunohistochemistry; IF, immunofluorescence; DAPI, 4',6-diamidino-2-phenylindole; PBS, phosphate-buffered saline Received 20.11.13; revised 12.1.14; accepted 10.2.14; Edited by P Salomoni
} 
misfolding accumulates during biosynthesis. The key component of the UPR, glucose regulated protein 78 (GRP78), dissociates from three types of ER stress initiators to lead to their activation due to viral infections, ischemia/reperfusion, hypoxia and other factors. ${ }^{17,18}$ These initiators include inositol requiring-1 $\alpha$ (IRE-1 $\alpha$ ), protein kinase RNA-like endoplasmic reticulum kinase (PERK) and activating transcription factor- $6 \alpha$ (ATF-6 $\alpha$ ), which promote correct folding and eliminate faulty protein. IRE- $1 \alpha$ has kinase and endoribonuclease (RNase) activities, whose autophosphorylation activates RNase activity to splice X-box binding protein 1 (sXBP1) mRNA, which produces the active transcription factor SXBP1. PERK phosphorylates eukaryotic initiation factor 2 alpha (elF2 $\alpha$ ), resulting in arrested protein translation. This arrest reduces the load entering the ER and concurrently selectively increases the translation of several mRNAs, which promotes the restoration of ER homeostasis. ATF- $6 \alpha$ transits to the Golgi compartment where it is cleaved by intra-membrane proteolysis to generate a soluble active transcription factor to affect protein folding and degradation. Endoplasmic reticulum protein 72 (ERp72), a member of the protein disulfide isomerase family, like GRP78, is also one of the ER chaperones and is the hallmark of UPR activation. Prolonged or severe ER stress impairs the protective mechanisms and ultimately activates caspase-12 or caspase- 4 to mediate apoptosis. $^{19-21}$

Mitochondrial apoptosis, as a classical death-signaling pathway, has an important role in ER stress-induced cell death. In addition, a significant fraction of endogenous Bcl-2 family proteins including Bax, Bak and PUMA have been shown to be associated with ER stress-induced mitochondrial apoptosis. $^{21}$ Although the function of PUMA in ER stress-mediated apoptosis has been extensively illustrated in different tissues, whether PUMA is involved in mucosal apoptosis in PHG remains still poorly understood, and whether PUMA induces PHG by mediating ER stress remains unclear.

In this study, we used a partial portal vein ligation (PVL)-induced PHG mouse model, a carbon tetrachloride $\left(\mathrm{CCl}_{4}\right)$-induced PHG mouse model and patient samples to study the role of PUMA-mediated apoptosis in PHG. We found that PUMA was induced by ER stress in PHG patients and mice PHG models. Targeted deletion of PUMA attenuated ER stress-induced gastric injury and mucosal epithelial apoptosis in PHG. These data suggest that PUMA has a pivotal role in PHG and mediates ER stress-induced mucosal epithelial apoptosis through the mitochondrial apoptotic pathway in PHG. The results indicate that PUMA is a potentially therapeutic target for PHG.

\section{Results}

PUMA was involved in PHG in humans. To study the role of PUMA in PHG patients and its associated gastric apoptosis, gastric mucosal specimens were analyzed in PHG patients without Helicobacter pylori infection and healthy volunteers. Histopathological analysis showed a loss of preserved architecture, edema and vasodilatation with inflammation cell infiltration in PHG mucosal samples compared with normal mucosa (Figure 1a). PUMA immunohistochemistry (IHC) staining showed that PUMA expression was markedly increased in PHG mucosal tissues compared with normal mucosa (Figure 1b). Although plentiful inflammatory cell infiltration was observed in mucosal tissues of PHG, only the gastric mucosal epithelium showed a significant expression of PUMA. Real-time PCR data showed that increased $P U M A$ expression was observed in the gastric mucosa of PHG and that PUMA mRNA expression increased 6-fold in the gastric mucosa of PHG patients compared with normal mucosa (Figures 1c and d). Furthermore, western blotting analysis showed that the expression of PUMA in $\mathrm{PHG}$ was significantly upregulated in the gastric mucosa compared with the normal tissues (Figures 1e and f). On the basis of these results, we conclude that PHG induces a significant gastric mucosal damage and PUMA expression and that PUMA is involved in PHG.

PHT induced gastric mucosal PUMA expression and PHG in PVL mice. We found that PUMA expression showed a significant upregulation in the mucosal tissue of PHG. However, it remained unknown whether PUMA expression was induced by PHT in animals. We established a portal hypertensive mouse model by PVL. After PVL for 2 weeks, the spleens in PVL mice showed significant megalosplenia and splenemia compared with sham-operated (SO) mice (Figure 2a). Histological analysis revealed little damage or inflammation in the gastric mucosa of SO mice but obvious inflammatory cell infiltration and destruction of the normal architecture with edema and vasodilatation were observed in the gastric mucosa of PVL mice (Figure 2b). To determine whether PUMA is involved in gastric mucosal injury in PVL mice, the gastric mucosal tissues from PHG and SO mice were examined. Immunofluorescence (IF) staining revealed that the gastric mucosal PUMA expression was remarkably higher in PVL mice compared with SO mice (Figure $2 b$ ). Real-time $\mathrm{PCR}$ revealed that the gastric mucosal PUMA mRNA level increased 5-fold in PVL mice compared with SO mice (Figures 2c and d). Consistent with the above findings, the mucosal PUMA protein level also showed a significant increase in PVL mice compared with SO mice (Figures $2 e$ and f). These results indicate that PHT induced gastric mucosal PUMA expression.

PHT enhanced gastric mucosal PUMA expression in $\mathrm{CCl}_{4}$-treated mice. Considering that $\mathrm{PHG}$ is a serious complication of liver cirrhosis following the classical PVL model, we then analyzed another independent PHG mouse model induced by cirrhosis to verify the role of PUMA in the pathogenetic mechanism of PHG. After intraperitoneal injection of $20 \% \mathrm{CCl}_{4}$ for 12 weeks in mice accompanied with little ascites, a large number of nodules were observed on the surface of the liver. Histopathological analysis showed that compared with vehicle-treated mice, the regular hepatic lobules in $\mathrm{CCl}_{4}$-treated mice disappeared and connective tissue hyperplasia formed pseudolobules in varying degrees of inflammatory cell infiltration (Figure 3a). These pathological alterations showed that liver cirrhosis has been induced in $\mathrm{CCl}_{4}$-treated mice. Similar to PHG mice from PVL-induced 
a
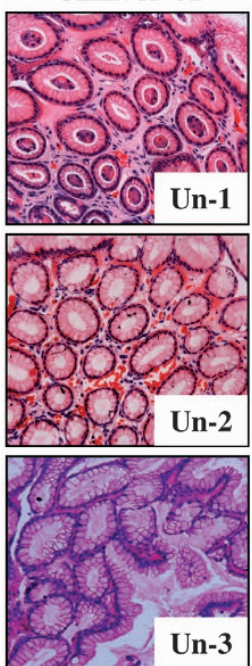

Un-3

$\mathrm{H} \& \mathrm{E}$

c

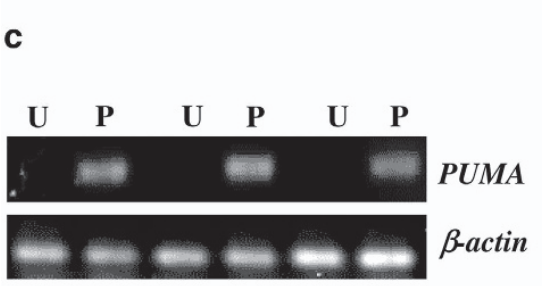

e

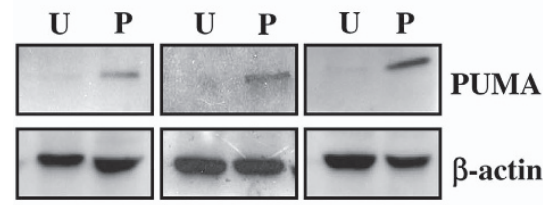

PHG
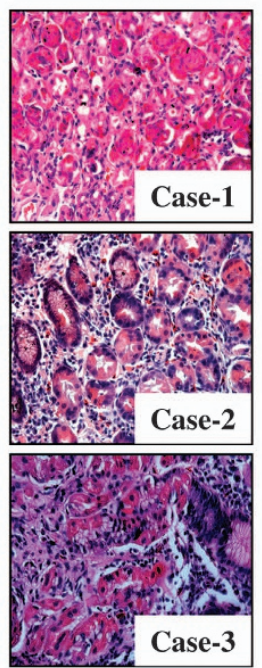

ase-3

-actin b
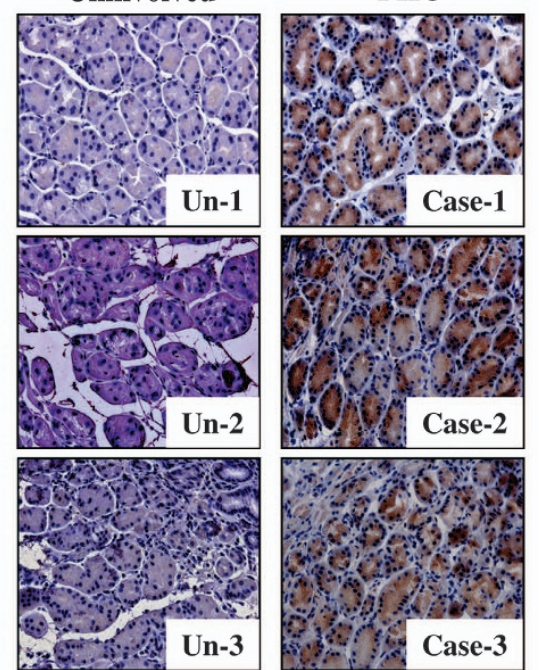

PUMA IHC
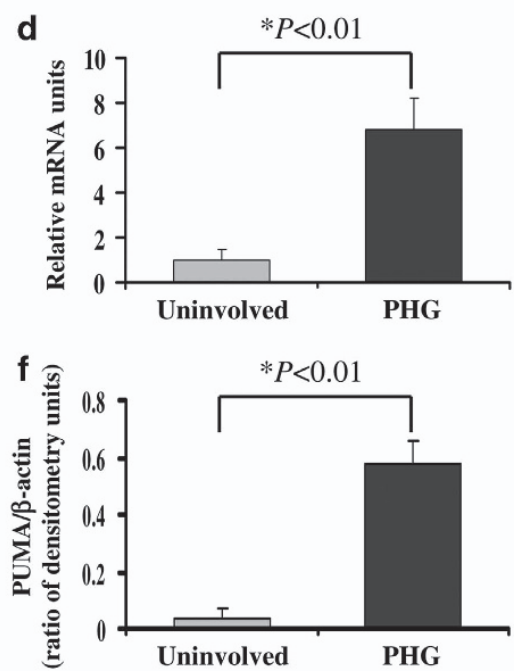

Figure 1 PUMA was involved in PHG in humans. (a) H\&E staining of uninvolved normal gastric mucosal tissues and gastropathic mucosal tissues from three PHG patients $(\times 200)$. (b) PUMA immunohistochemistry $(\mathrm{IHC})$ staining (brown) of uninvolved normal gastric mucosal tissues and gastropathic mucosal tissues from three PHG patients $(\times 200)$. (c) PUMA mRNA expression in the gastric mucosa was evaluated by real-time PCR in three pairs of different specimens. U: uninvolved normal gastric mucosa, P: PHG mucosa. (d) Real-time PCR showed a relative PUMA mRNA fold change in uninvolved normal gastric mucosa and PHG gastric mucosa. The values are expressed as the means \pm S.D. ( $n=3$ in each group) for real-time PCR. (e) PUMA protein expression in the gastric mucosa was determined by western blotting in three pairs of different specimens. $\beta$-Actin was used as the loading control. U: uninvolved normal gastric mucosa, P: PHG mucosa. (f) The ratio of densitometry units of PUMA/ $\beta$-actin is represented. The values are expressed as the means \pm S.D. ( $n=3$ in each group) for western blotting

model, liver cirrhosis-induced PHT also caused gastric mucosal damage with an inflammatory response. PUMA expression of the gastric mucosal tissues was significantly higher in $\mathrm{CCl}_{4}$-treated mice compared with vehicle-treated mice (Figure $3 b$ ). Real-time PCR showed that the gastric mucosal PUMA mRNA expression was significantly increased (Figures $3 \mathrm{c}$ and $\mathrm{d}$ ), and the mucosal PUMA protein level was also markedly upregulated in $\mathrm{CCl}_{4}$-treated mice compared with vehicle-treated mice (Figures $3 e$ and f). These results further indicate that similar to the samples from PHG patients and PVL mouse model, PUMA is substantially required in $\mathrm{PHT}$-induced gastric injury to lead to the development of $\mathrm{PHG}$ in the $\mathrm{CCl}_{4}$-treated liver cirrhosis model.
PUMA-mediated epithelial apoptosis contributed to PHG. To investigate the effect of PUMA on PHG, we next analyzed whether PUMA-mediated mucosal apoptosis contributes to PHG. The terminal deoxynucleotidyl transferase-mediated deoxyuridine triphosphate nick end labeling (TUNEL) assay showed that evident gastric mucosal apoptosis was found in PHG patients, and double staining of TUNEL and PUMA indicated that PUMA is involved in mucosal apoptosis in PHG (Figure 4a). Furthermore, we labeled gastric mucosal epithelial cells with cytokeratin staining which is a marker of epithelial cells, double staining of TUNEL and cytokeratin demonstrated that the apoptotic cells were mucosal epithelium in PHG (Figure 4a). In PVL mice, gastric mucosal epithelial apoptosis was significantly 
a

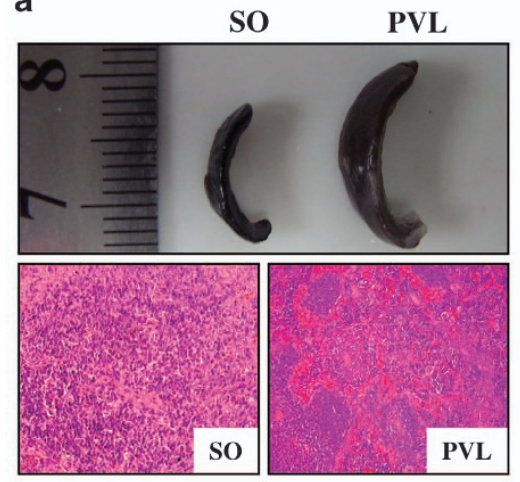

c

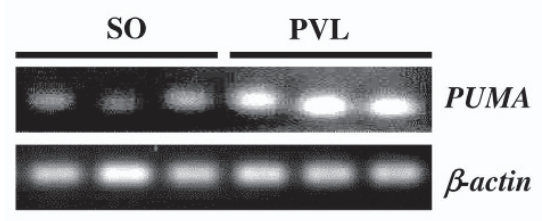

e

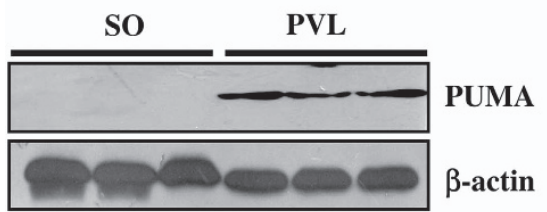

b

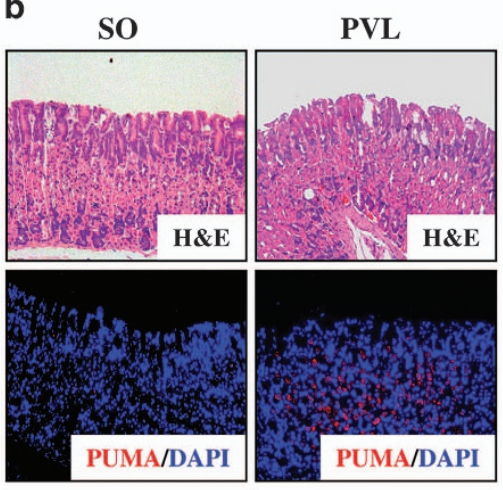

d

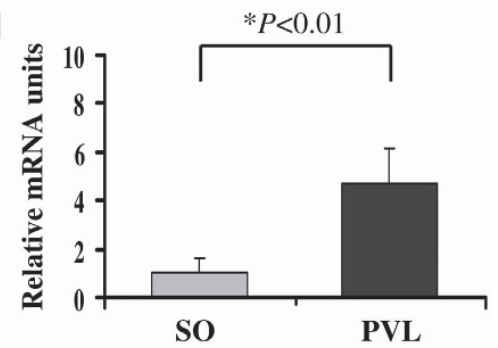

f

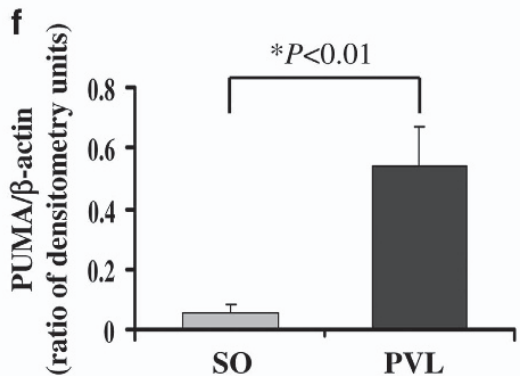

Figure 2 Portal hypertension induced gastric mucosal PUMA expression and PHG in PVL mice. (a) Portal hypertension induced significant congestive splenomegaly, and H\&E staining showed splenemia with splenitis in PVL mice at 2 weeks compared with SO mice $(\times 200)$. (b) Portal hypertension significantly induced gastric mucosal injury (upper panel H\&E staining, $\times 200$ ) and upregulated PUMA (red) expression (lower panel immunofluorescence staining, $\times 200$ ) in PVL mice. Cell nuclei (blue) were counterstained by 4',6-diamidino-2-phenylindole (DAPI). (c) PUMA mRNA expression in the gastric mucosa was evaluated by real-time PCR in three different mice in each group. (d) Real-time PCR showed relative PUMA mRNA fold changes. The values are expressed as the means \pm S.D. ( $n=3$ in each group) for real-time PCR. (e) PUMA protein expression in the gastric mucosa was determined by western blotting in three different mice. $\beta$-Actin was used as the loading control. (f) The ratio of densitometry units of PUMA/ $\beta$-actin is represented. The values are expressed as the means \pm S.D. ( $n=3$ in each group) for western blotting

increased, and PUMA expression was also markedly upregulated compared with SO mice (Figure 4b). Further, marked gastric mucosal epithelial apoptosis and higher PUMA expression were synchronously observed in $\mathrm{CCl}_{4}$ treated mice but not in vehicle-treated mice (Figure 4c). These data suggest that PUMA is involved in gastric mucosal epithelial apoptosis in PHG.

Targeted deletion of PUMA attenuated PHG by inhibiting epithelial apoptosis. To confirm PUMA-mediated gastric mucosal epithelial apoptosis in PHG, we analyzed the effect of PUMA using PUMA-KO mice in a PVL model. The genotyping of mice was determined by PCR (Figure $5 \mathrm{a}$ ). After PVL for 2 weeks, the spleens in PVL mice showed significant megalosplenia and splenemia compared with SO mice; however, no significant difference between PUMA-WT and PUMA-KO mice was observed (Figure 5a). Pathological analysis showed that in PVL mice, marked gastric mucosal damage with mucosal erosion and edema was observed compared with SO mice; however, the damage was significantly attenuated in the PUMA-KO mice (Figure $5 \mathrm{~b}$ ). These results demonstrated that the targeted deletion of PUMA relieved the gastric damage induced by PHG but did not reduce PHT. To further confirm the effect of PUMA on mucosal apoptosis in PHG, TUNEL staining was performed to determine the location and percentage of the apoptotic cells in situ. Few TUNEL-positive cells were detected in the gastric mucosa in SO mice from both PUMA-WT and PUMA-KO mice; however, in PVL mice, the number of apoptotic cells was found in the gastric mucosa (Figure 5c). Apoptosis was significantly attenuated in the PUMA-KO mice with the apoptotic index reduced by over $50 \%$ following PVL (Figures $5 \mathrm{c}$ and $\mathrm{d}$ ). These results demonstrated that PUMA is an important mediator in PHG.

PUMA-mediated apoptosis depended on mitochondrial apoptotic signaling in PHG. Above these results have demonstrated that PUMA is an important mediator in 
a

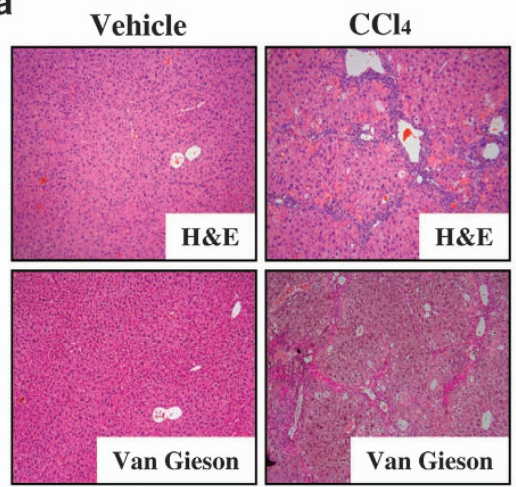

C

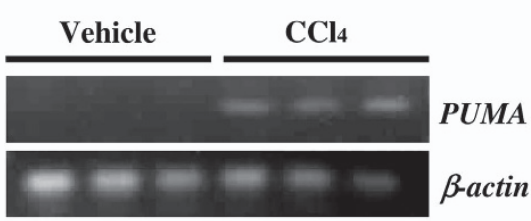

e

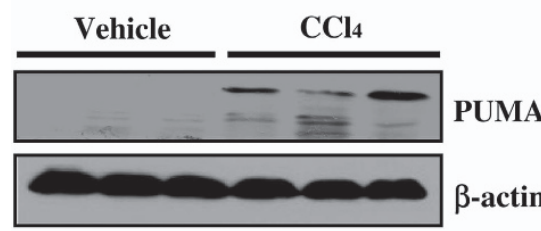

b

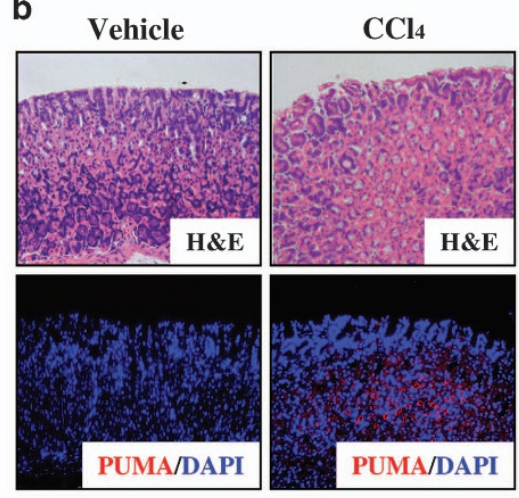

d
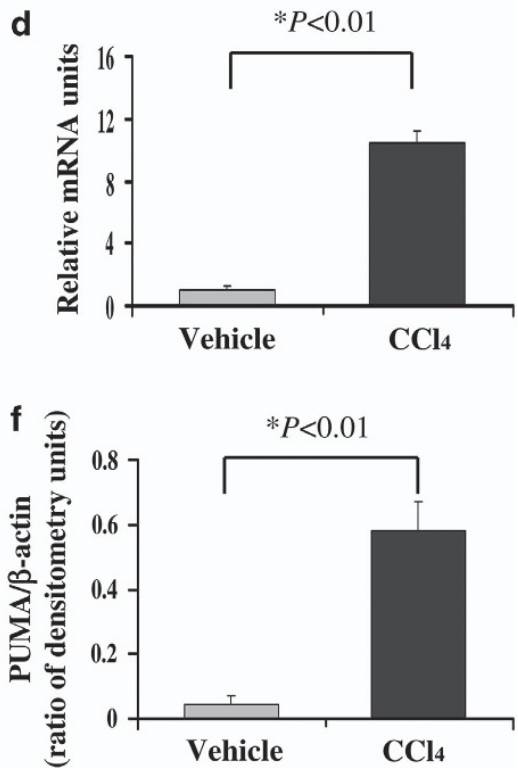

Figure 3 Portal hypertension enhanced gastric mucosal PUMA expression in $\mathrm{CCl}_{4}$-treated mice. (a) Histological analysis showed that $\mathrm{CCl}_{4}$ induced significant liver cirrhosis. The upper panel is H\&E staining $(\times 200)$, and the lower panel is Van Gieson staining $(\times 200)$. (b) Portal hypertension significantly induced gastric mucosal injury (upper panel H\&E staining, $\times 200$ ) and upregulated PUMA (red) expression (lower panel immunofluorescence staining, $\times 200$ ) in mice after $\mathrm{CCl}_{4}$ intraperitoneal injection for 12 weeks. Cell nuclei (blue) were counterstained by DAPI. (c) PUMA mRNA expression in the gastric mucosa was evaluated by real-time PCR in three different mice in each group. (d) Real-time PCR showed a relative PUMA mRNA fold change. The values are expressed as the means \pm S.D. $(n=3$ in each group) for real-time PCR. (e) PUMA protein expression in the gastric mucosa was determined by western blotting in three different mice. $\beta$-Actin was used as the control for loading. (f) The ratio of densitometry units of PUMA/ $\beta$-actin is represented. The values are expressed as the means \pm S.D. ( $n=3$ in each group) for western blotting

PHT-induced gastric mucosal injury and apoptosis. PUMA is one of the most potent mediators that transduce death signals primarily to mitochondrion. During mitochondriamediated apoptosis, several mitochondrial pro-apoptotic proteins, such as cytochrome $c$, are released into the cytosol to facilitate the formation of the apoptosome and subsequent activation of the caspase cascade. ${ }^{22}$ Consistent with the above results, in the PVL mouse model, PUMA-positive cells and apoptotic cells were noticeably increased in the gastric mucosa after PVL (Figure 6a). To investigate the mechanisms of PHT-induced and PUMA-mediated apoptosis, mitochondrial and cytosolic fractions were purified through differential centrifugation from the mucosa of the SO and PVL animals (Figure 6b). The release of cytochrome $c$ was examined by western blotting. In the SO mice, cytochrome $c$ was detected in the mitochondrial fractions but not in the cytosolic fractions. The amount of cytochrome $c$ was markedly increased in the cytosolic fractions of the gastric mucosa after PVL. Concurrently, Bax mitochondrial translocation and Bak alteration were also found to be induced in the PVL mouse model. As the subsequent executors of apoptosis of the mitochondrial pathway, cleaved caspase- 9 and cleaved caspase-3 were also significantly increased after PVL for 2 weeks in mice (Figures $6 \mathrm{c}$ and d). To further explore whether this situation also occurs in the gastric mucosa of PHG patients, human PHG mucosal tissue samples were purified to investigate the induction of apoptotic executors. Western blotting data revealed that the activation of caspase- 9 and caspase- 3 significantly increased in PHG mucosal tissues but not in uninvolved gastric mucosal tissues (Figures $6 e$ and f). To summarize, these results suggest that alterations in Bax and Bak, 


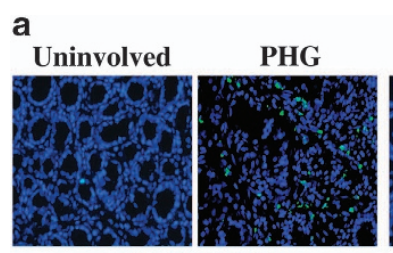

TUNEL/DAPI

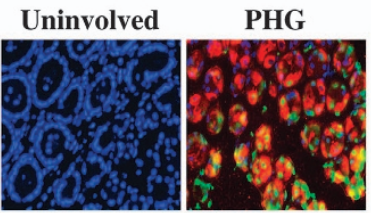

PUMA/TUNEL/DAPI

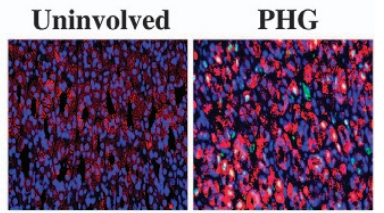

Cytokeratin/TUNEL/DAPI

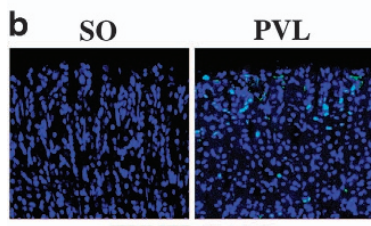

TUNEL/DAPI

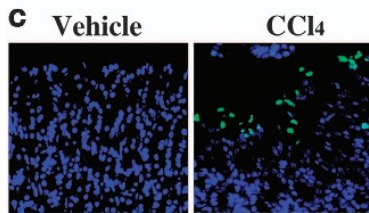

TUNEL/DAPI
So

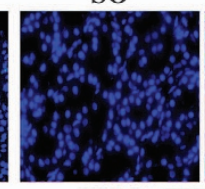

PUMA/TUNEL/DAPI

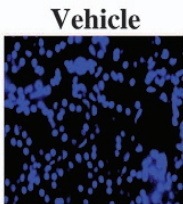

PUMA/TUNEL/DAPI
PVL

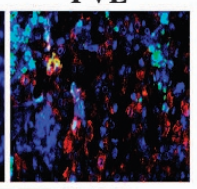

$\mathrm{CCl}_{4}$

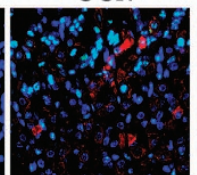

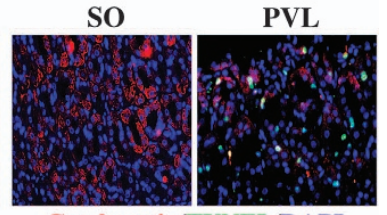

Cytokeratin/TUNEL/DAPI

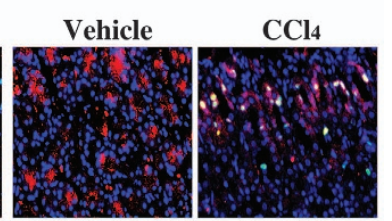

Cytokeratin/TUNEL/DAPI

Figure 4 PUMA-mediated apoptosis contributed to PHG. Apoptotic cells (green) were detected by TUNEL staining $(\times 400)$, and PUMA (red) and cytokeratin (red) expression was determined by immunofluorescence staining $(\times 400)$ in the gastric mucosal tissues. Cell nuclei (blue) were counterstained by DAPI $(\times 400)$. (a) Gastric mucosal specimen of PHG patients. (b) Gastric mucosal specimen of PVL mice. (c) Gastric mucosal specimen of $\mathrm{CCl}_{4}$-treated mice

a

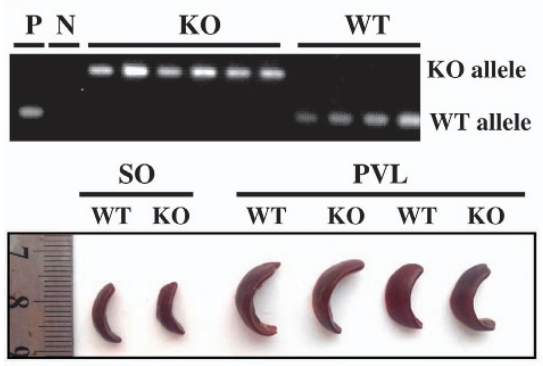

c

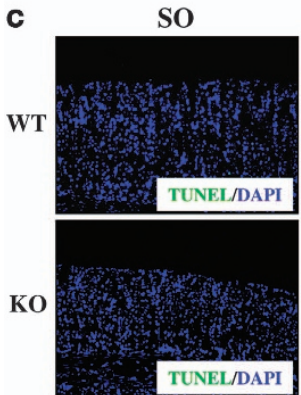

b

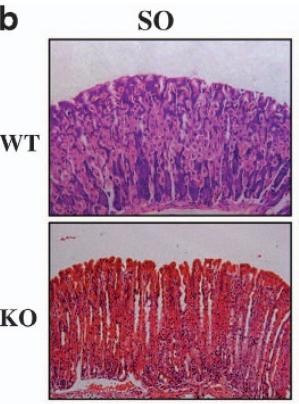

d

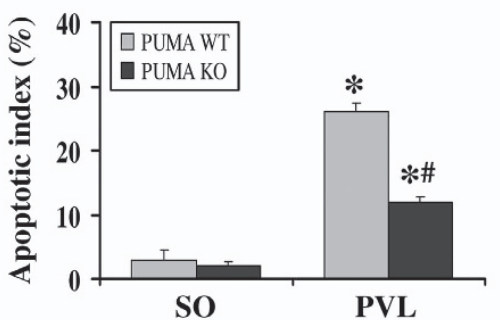

Figure 5 Targeted deletion of PUMA attenuated PHG by inhibiting apoptosis. (a) Genotyping of mice was performed by using PUMA allele-specific primers to analyze

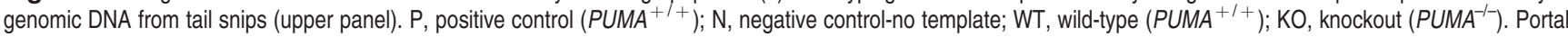
hypertension induced significant congestive splenomegaly after PVL in both PUMA-WT and KO mice (lower panel). (b) Gastric mucosal injury was significantly alleviated in PUMA-KO mice after PVL compared with PUMA-WT mice (H\&E staining, $\times 200$ ). (c) Gastric mucosal apoptosis (green) was significantly depressed in PUMA-KO mice after PVL compared with PUMA-WT mice (TUNEL staining, $\times 200$ ). Cell nuclei (blue) were counterstained by DAPI. (d) The apoptotic index was calculated by counting a minimum of 20 randomly selected fields following TUNEL staining. The index was obtained by dividing the TUNEL-positive cells by the total number of cells. The values are expressed as the means \pm S.D. ( $n=3$ in each group). ${ }^{*} P<0.01$ versus $S 0$ mice, ${ }^{\#} P<0.05$ versus $P U M A-W T$ mice

cytochrome $c$ release, caspase- 9 , and caspase- 3 activation were involved in PUMA-mediated gastric mucosal apoptosis in PHG.
ER stress-activated mitochondrial apoptotic signaling by PUMA-induced mucosal apoptosis in PHG. Although our above results have demonstrated that PUMA was 


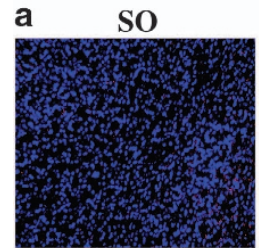

PUMA/DAPI

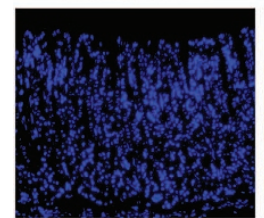

TUNEL/DAPI

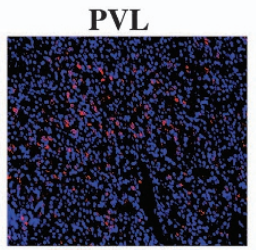

PUMA/DAPI

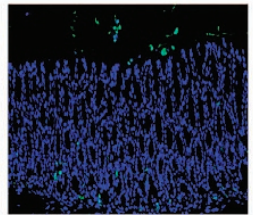

TUNEL/DAPI
C

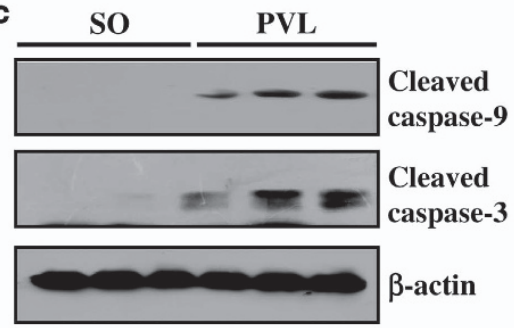

e

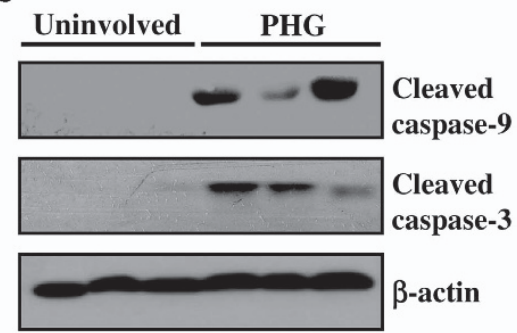

b Cytosolic Mitochondrial

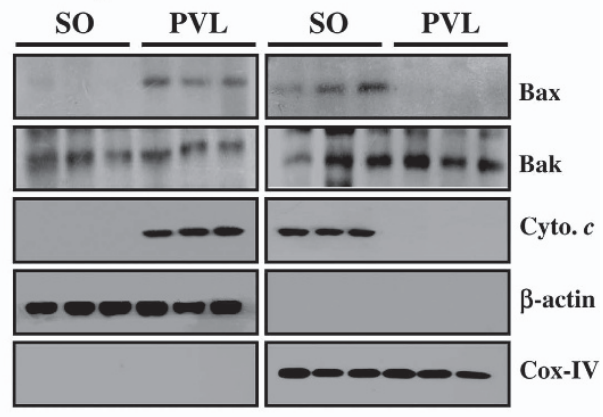

d

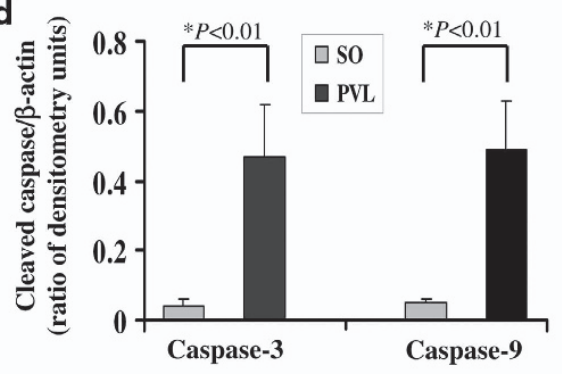

f

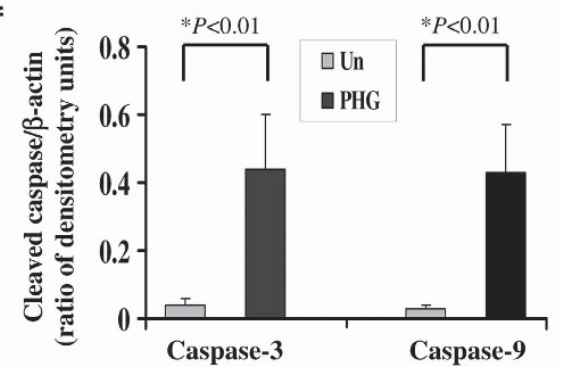

Figure 6 PUMA-mediated apoptosis depended on mitochondrial apoptotic signaling in PHG. (a) Portal hypertension significantly induced PUMA (red) expression and mucosal apoptosis (green) in gastric mucosal tissues of PVL mice. Cell nuclei (blue) were counterstained by DAPI $(\times 200)$. (b) Mitochondrial and cytosolic fractions were analyzed for Bax, Bak and cytochrome $c$ by western blotting ( $n=3$ in each group). $\beta$-Actin and Cox IV were markers of cytosolic and mitochondrial fractions, respectively. (c and e) Gastric mucosal cleaved caspase- 9 and cleaved caspase-3 expression was evaluated by western blotting in both PVL mice and PHG patients ( $n=3$ in each group). $\beta$-Actin was used as the loading control. ( $\mathbf{d}$ and $\mathbf{f}$ ) The ratio of densitometry units of cleaved caspase- $9 / \beta$-actin and cleaved caspase- $3 / \beta$-actin was represented in both PVL mice and PHG patients. The values are expressed as the means \pm S.D. ( $n=3$ in each group) for western blotting

essential for gastric mucosal apoptosis, and the PUMAmediated apoptosis is mitochondria dependent in PHG, it remained unclear whether $E R$ stress induced gastric mucosal apoptosis via PUMA in PHG. Furthermore, we investigated whether ER stress activates mitochondrial apoptotic signaling by PUMA in mucosal apoptosis of PHG. GRP78, commonly used as a marker of ER stress, is a key element of the trigger for all UPR downstream pathways. ${ }^{19}$ The analysis of GRP78 by western blotting in PUMA-WT mice revealed that GRP78 expression was markedly induced in the gastric mucosa of PVL mice, in addition, the expression of several of its downstream substrates such as sXBP-1 and phosphorylated elF2 $\alpha$ (p-elF $2 \alpha$ ) was distinctly upregulated following GRP78 induction, and caspase-12, an ER-localized caspase, was also distinctly activated in PVL mice (Figure 7a). Furthermore, the result of $\mathrm{IHC}$ staining showed that gastric mucosal GRP78 and cleaved caspase-12 were significantly increased in PVL mice compared with SO mice (Figure 7c). These results suggested that ER stress involved in $\mathrm{PHG}$ in response to PHT.

Although mucosal injury and apoptosis in PVL-induced PHG have been demonstrated to be alleviated by targeted deletion of PUMA, the PUMA-KO did not repress PVL-induced GRP78 and cleaved caspase-12 expression; however, targeted deletion of PUMA significantly inhibited the activation of caspase-9 and caspase-3 (Figure 7b). Furthermore, IF double staining of GRP78 and PUMA suggested that PUMA was a mediator of ER stress in PVL-induced PHG (Figure 7d). Moreover, using a $\mathrm{CCl}_{4}$-induced PHG mouse model, we found that the results were in accordance with the PVL-induced PHG model (data not shown). In the gastric mucosal specimens of PHG patients, GRP78, sXBP1 and p-elF2 $\alpha$ were remarkably upregulated, and caspase- 4 was 

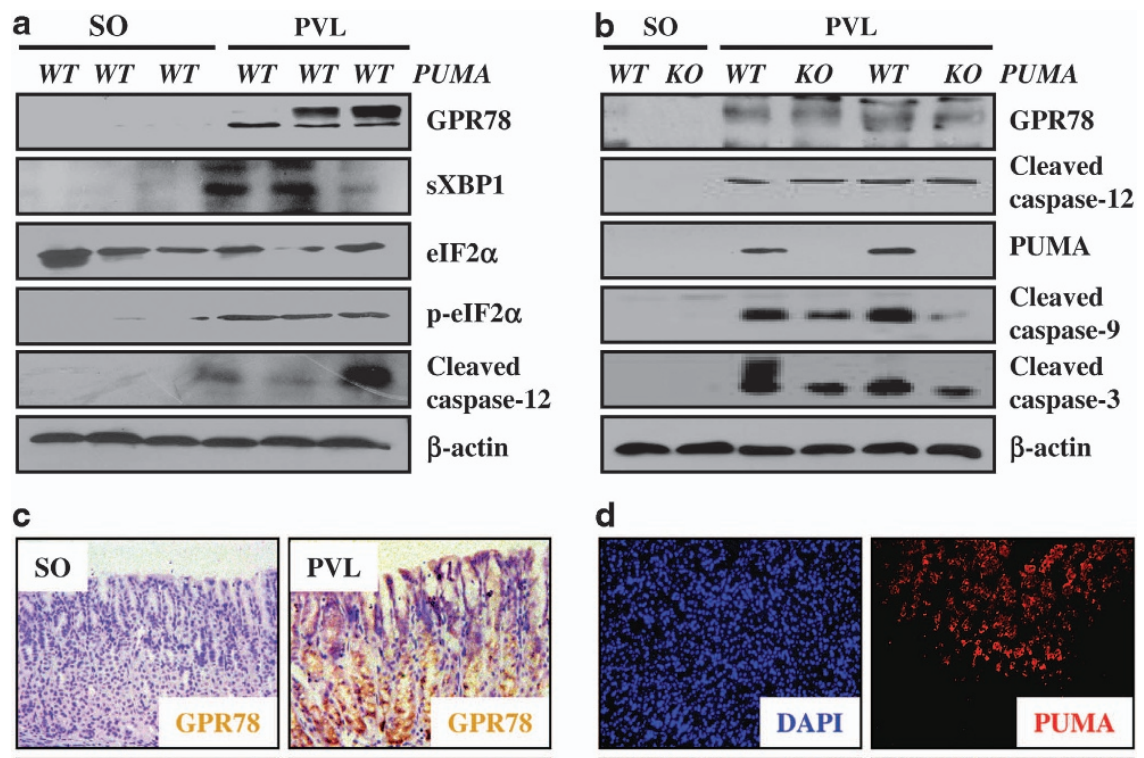

d
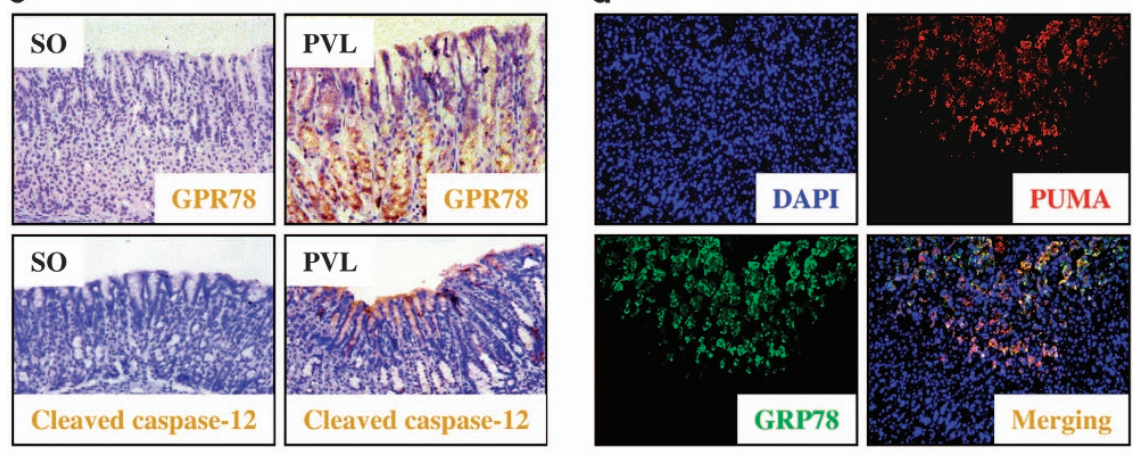

e

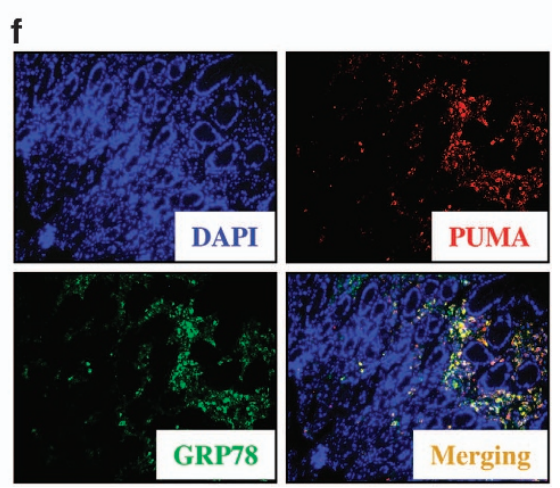

Figure 7 ER stress-activated mitochondrial apoptotic signaling by PUMA induced mucosal apoptosis in PHG. (a) PVL induced significant ER stress signaling in mice. Three mice were used in each group. $\beta$-Actin was used as the loading control. (b) Targeted deletion of PUMA significantly repressed ER stress-induced caspase-9 and caspase-3 activation by PVL in mice. (c) Immunohistochemistry staining showed that the expression of GRP78 and cleaved caspase-12 was induced by PVL in mice (brown, $\times 200$ ). (d) Double immunofluorescence staining of GRP78 and PUMA indicated that PUMA upregulation followed GRP78 induction in the gastric mucosa of PVL mice $(\times 200)$. (e) ER stress signaling pathway was activated in the gastric mucosa of PHG patients. $\beta$-Actin was used as the loading control. (f) Double immunofluorescence staining of GRP78 and PUMA also confirmed that PUMA upregulation followed GRP78 induction in the gastric mucosa of human PHG $(\times 200)$

dramatically activated compared with the uninvolved gastric mucosal specimens (Figure 7e). IF double staining of GRP78 and PUMA also indicted that PUMA was an apoptotic mediator in response to ER stress in human PHG (Figure 7f). These results suggest that $E R$ stress activated mitochondrial apoptotic signaling by PUMA to induce mucosal apoptosis in PHG.

PUMA responded to ER stress-induced apoptosis in vitro. To investigate mechanism of PUMA mediated ER stress-induced apoptosis, two gastric cell lines GES-1 and SGC7901 were used in this study. GES-1 cells and SGC7901 cells were treated with tunicamycin, which blocks ER protein glycosylation to induce ER stress. Following the treatment of tunicamycin, GRP78 expression was observably upregulated after tunicamycin treatment for $4 \mathrm{~h}$ in GES-1 cells and SGC7901 cells, and reached maximal levels at 24 and $16 \mathrm{~h}$, respectively. Furthermore, nearly the same trend was observed in ER-localized caspase and cleaved caspase-4, PUMA and cleaved caspase-3 were also induced in both of the two types of cells (Figure 8a). We further analyzed cellular apoptosis by ER stress inducer tunicamycin using the TUNEL assay. After tunicamycin treatment for $24 \mathrm{~h}$, cellular apoptosis was observed in the two cell lines, and the apoptotic index increased to $\sim 41$ and $26 \%$, respectively (Figures $8 \mathrm{~b}$ and $\mathrm{c}$ ).

To further investigate whether PUMA responded to ER stress-induced apoptosis in gastric cells lines, we used small 
a

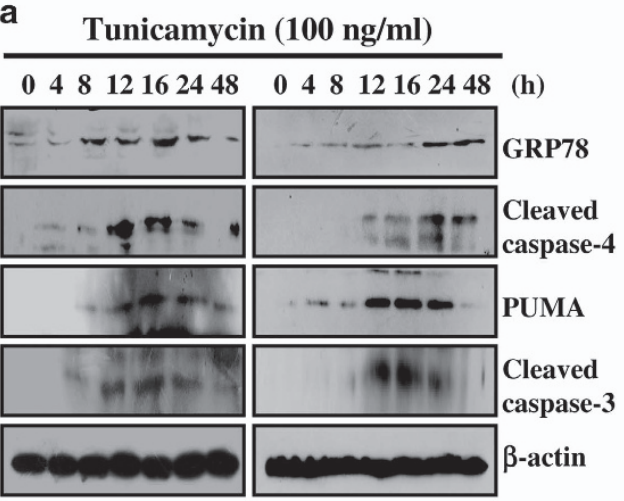

GES-1

SGC7901

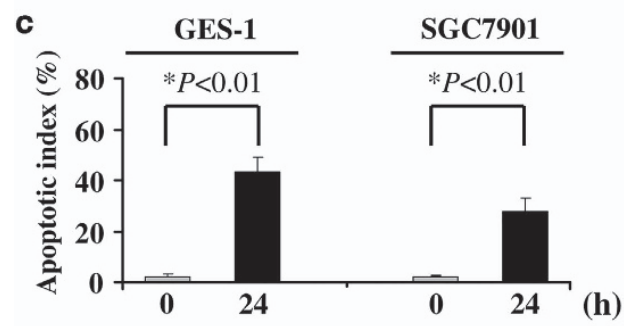

b

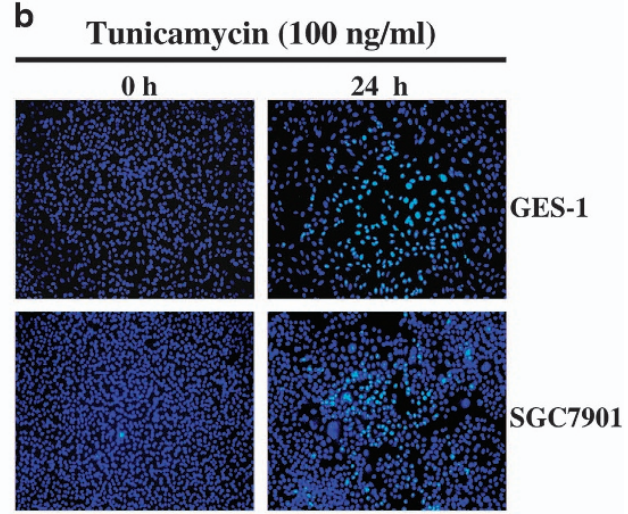

TUNEL/DAPI

d

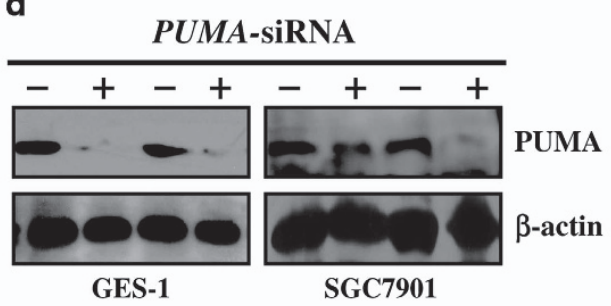

e Tunicamycin $(100 \mathrm{ng} / \mathrm{ml})$

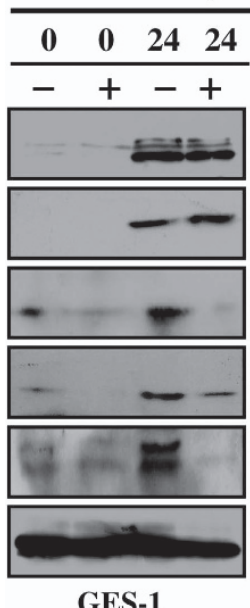

$\begin{array}{llllll}0 & 0 & 24 & 24 & \text { (h) }\end{array}$

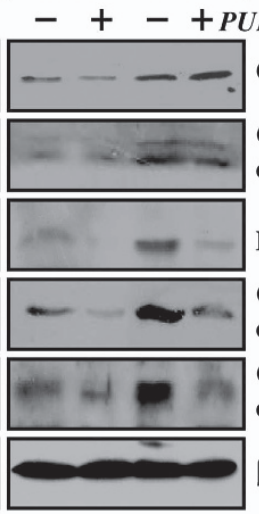

SGC7901 f

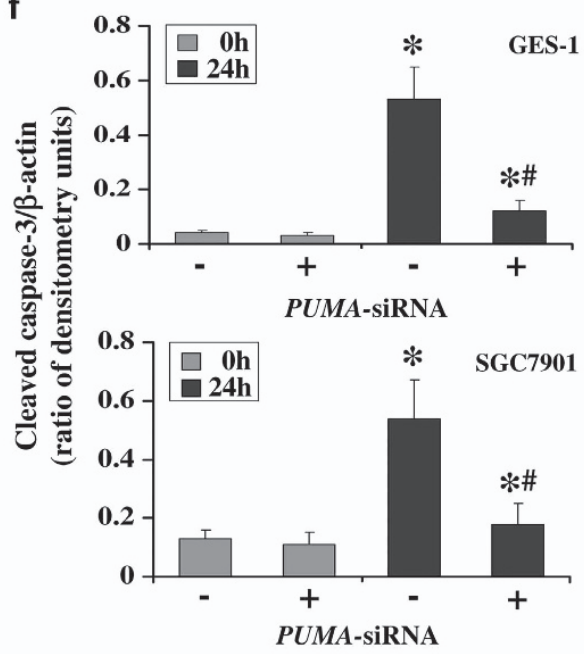

Figure 8 PUMA mediated ER stress-induced apoptosis in vitro. (a) GES-1 and SGC7901 cells were treated with the ER stress inducer tunicamycin, and the western blotting assay showed that GRP78 and cleaved caspase-4 were significantly induced, and PUMA and cleaved caspase-3 were markedly upregulated. $\beta$-Actin was used as the loading control. (b) TUNEL (green) staining showed that tunicamycin remarkably induced apoptosis in GES-1 and SGC7901 cell lines. Cell nuclei (blue) were counterstained by DAPI $(\times 200)$. (c) The apoptotic index was calculated by counting a minimum of 20 randomly selected fields following TUNEL staining. The index was obtained by dividing the TUNEL-positive cells by the total number of cells. (d) Western blotting showed that PUMA-siRNA significantly knocked down PUMA expression in GES-1 and SGC7901 cell lines. (e) PUMA-siRNA did not affect GRP78 expression and caspase-4 activation after tunicamycin treatment, however, PUMA knocked down evidently inhibited ER stress-induced caspase-9 and caspase-3 activation by tunicamycin. (f) The ratio of densitometry units of cleaved caspase-3/ $\beta$-actin was represented in two cell lines. The values are expressed as the means \pm S.D., and the values were achieved by three separate experiments. ${ }^{*} P<0.01$ versus $0 \mathrm{~h},{ }^{\#} P<0.01$ versus $24 \mathrm{~h}$ without PUMA-siRNA treatment

interfering RNA (siRNA) to suppress PUMA expression. Two siRNAs were purchased and tested for anti-PUMA activity, and ultimately, one of them was selected based on its ability to knockdown PUMA (Figure 8d). We next examined whether PUMA responded to ER stress-induced apoptosis. On the basis of the above data, we singled out one time point, at $24 \mathrm{~h}$ after tunicamycin, to analyze whether this PUMA-siRNA was able to effectively suppress the apoptosis inducted by tunicamycin. PUMA knockdown did not affect the expression of GPR78 and cleaved caspase-4 after tunicamycin treatment (Figure 8e). However, the inhibition of PUMA significantly suppressed its downstream pro-apoptotic elements such as caspase- 9 and caspase- 3 activation (Figures $8 \mathrm{e}$ and $\mathrm{f}$ ). In summary, the PUMA knockdown evidently protected these 
cells from apoptosis induced by ER stress, which provided evidence that PUMA is a critical mediator of ER stressinduced apoptosis.

\section{Discussion}

In this study, the gastric mucosa from PHG patients showed a destruction of architecture, edema with erosion, vasodilatation with lymphocyte infiltration and significant apoptosis induction compared with the uninvolved normal mucosa. Furthermore, PUMA expression was significantly increased, and a number of apoptotic cells were observed in the gastric mucosa of PHG patients. The results indicated that PUMA has an essential role in PHG. Several studies have revealed PHG improvement after transjugular intrajugular intrahepatic portosystemic shunt (TIPS) and shunt surgery, suggesting an association between PHG and the severity of PHT; however, others have failed to show this linear correlation with the severity of PHT. ${ }^{23,24}$ On the basis of these findings, we used two types of PHG mouse models to explore the effect of PUMA on PHG, which were PVL- and $\mathrm{CCl}_{4}$-induced PHG in mice. Our previous study demonstrated that PVL induced evident PHG after PVL for 2 weeks, ${ }^{7}$ and another report showed that $\mathrm{CCl}_{4}$ induced prominent $\mathrm{PHG} .{ }^{25}$ In the study, the results showed that two mouse models led to significant $\mathrm{PHT}$ and PHG, upregulated gastric mucosal PUMA expression and induced mucosal epithelial apoptosis. The animal model data were consistent with human PHG. Furthermore, our experiment demonstrated that the PUMA deficiency remarkably attenuated PVL- and $\mathrm{CCl}_{4}$-induced PHG and gastric mucosal epithelial apoptosis. Our study suggested that PUMA is involved in PHG by inducing epithelial apoptosis. PUMA, a BH3-only Bcl-2 family protein, has an essential role in p53-dependent and p53-independent apoptosis, and the BH3-only Bcl-2 family members such as Noxa, Bid and Bim regulate apoptosis through multiple redundant and parallel pathways. $^{26,27}$ Potentially, several $\mathrm{BH} 3$-only proteins are collectively responsible for apoptosis in PHG.

PUMA is normally expressed at a very low level but is rapidly induced in response to a wide range of stimuli in different tissues, and after induction, PUMA transduces death signals to the mitochondria, where it activates the multi-domain proapoptotic proteins Bax and Bak to trigger mitochondrial dysfunction and caspase activation. ${ }^{14,28}$ Mitochondria have a key role in cell life and death. Several mitochondrial apoptogenic proteins, including cytochrome $c$, are released into the cytosol to initiate apoptosis through the formation of the apoptosome and subsequent activation of the caspase cascade. Our previous study authenticated that PUMA mediates the ischemia/reperfusion-induced intestinal apoptosis via the mitochondria apoptotic pathway. ${ }^{29}$ In this study, our data indicated that PUMA-mediated gastric mucosal epithelial apoptosis in PHG via its regulation of Bax/Bak, cytochrome $c$ release and caspase activation. The mitochondrial pathway of apoptosis requires the release of cytochrome $c$ from the mitochondrion to the cytosol. Once released, cytochrome $c$ cooperates with the adaptor protein, pro-caspase-9, to promote the activation of caspase-3, which is the apoptotic executor leading to cell death. PUMA acts through the proapoptotic multi-domain Bcl-2 effector proteins,
Bax and Bak, which oligomerize into proteolipid pores and permeate the outer membrane of the mitochondrion to allow the efflux of cytochrome $c$ and other intermembrane space proteins to the cytosol to induce apoptosis. ${ }^{11,30,31}$ These data demonstrated that PUMA mediated gastric mucosal epithelial apoptosis through mitochondria-dependent apoptotic signaling in PHG.

PUMA mediates cell apoptosis by a variety of stimuli including ER stress. ER stress is a phenomenon that occurs when excessive protein misfolding occurs during biosynthesis. ER stress triggers a series of signaling and transcriptional events known as UPR. The UPR attempts to restore homeostasis in the ER but prolonged or severe UPR triggers apoptosis in the stressed cells. ${ }^{19}$ Gastric cells are susceptible to ER stress because they produce large amounts of secretive proteins such as PGE2 and pepsinogen, which are involved in mucosal defense and digestion, respectively. ${ }^{32}$ $P U M A$ is identified as an ER stress-responsive gene in global gene expression, and PUMA is able to mediate ER stressinduced apoptosis in a variety of cell types. ${ }^{33,34}$ The induction of PUMA in response to ER stress is p53 independent in most cases. ${ }^{15,35,36}$

To confirm PUMA as an apoptotic mediator by induction of ER stress, we used the disulfide bond inhibitor tunicamycin, which induces the accumulation of misfolded proteins and ER stress to stimulate two types of gastric epithelial cell lines, GES-1 and SGC7901, then explored the effect of PUMA on ER stress-induced apoptosis. After tunicamycin treatment, GRP78 and cleaved caspase-4 were markedly induced, PUMA expression and caspase-3 activation were dramatically upregulated, and apoptosis was significantly triggered in both GES-1 and SGC7901 cell lines. Furthermore, we knocked down PUMA by PUMA-siRNA and found that the expressions of GRP78 and cleaved caspase-4 were not repressed. However, the activation of caspase- 9 and caspase-3 was significantly inhibited in the two cell lines. The results affirmed that PUMA mediates ER stress-induced apoptosis. In addition, we also found that the PUMA deficiency significantly ameliorated ER stress-induced mucosal apoptosis in PHG using PUMA-KO mice. In PHG patient samples, we confirmed that PUMA is involved in ER stressinduced mucosal apoptosis. Overall, the data provided strong evidence to suggest that PUMA mediates ER stress-induced mucosal apoptosis in PHG.

In summary, PHT triggered gastric mucosal ER stress, the ER stress upregulated PUMA expression, and PUMA significantly mediated mucosal epithelial apoptosis via the mitochondria-dependent apoptotic pathway. The results suggest that PUMA mediates ER stress-induced mucosal injury and epithelial apoptosis in PHG, and indicates that PUMA is a potentially therapeutic target for PHG.

\section{Materials and Methods}

Tissue samples. Frozen gastric mucosal specimens of 10 PHG without $H$. pylori infection and 10 uninvolved healthy volunteers were obtained from Digestive Disease Tissue Resource of The Third Affiliated Hospital of Sun Yat-Sen University. The acquisition of tissue samples was approved by the Institutional Review Board at The Third Affiliated Hospital of Sun Yat-Sen University. Written informed consent was received from each patient and healthy volunteers before inclusion in the study. 
Animals and induction of PHT. All animal experiments were approved by the Institutional Animal Care and Use Committee at Sun Yat-Sen University. Eight- to ten-week-old male mice $(20-25 \mathrm{~g})$ were used for all experiments. The mice were housed in microisolator cages and allowed access to water and chow ad libitum. PUMA ${ }^{+/}$mice (Jackson Laboratory, Bar Harbor, ME, USA) in the C57BL/6 background were used to generate PUMA ${ }^{+/+}$and $P U M A^{-/}$littermates. The mice were housed in microisolator cages in a room illuminated from 0800 to $2000 \mathrm{~h}(12: 12 \mathrm{~h} \mathrm{light-dark}$ cycle) and were allowed free access to water and food. The mice were genotyped by PCR using genomic DNA extracted from tail snips as previously described. ${ }^{29}$ PHT was induced by a portal vein constriction as previously described. ${ }^{7}$ A laparotomy was performed under halothane anesthesia. The portal vein was isolated, and calibrated constriction was performed using a single ligature of 3-0 silk around the portal vein and a 27-gauge blunt-tipped needle. The needle was then removed, leaving a calibrated stenosis of the porta vein. This technique involved the initial constriction of the portal vein to $\sim 50 \%$ of its diameter. In SO mice, the same operation was performed with the exception that no ligature was placed after isolating the portal vein. After the operation, the animals were housed in cages and allowed free access to food and water until the experiments were performed. The liver cirrhosis model with PHT was established by an intraperitoneal injection of $20 \% \mathrm{CCl}_{4}$ (Sinopharm Chemical Reagent, Shanghai, China) dissolved in an olive oil solution (Sinopharm Chemical Reagent) at $5 \mathrm{ml} / \mathrm{kg}$ body weight, twice per week for 8 weeks. The vehicle group was only intraperitoneally injected with olive oil, at $5 \mathrm{ml} / \mathrm{kg}$ body weight, twice per week for 8 weeks.

Samples collection and preparation. After PHT was induced from the two mouse models, the animals were anesthetized and then euthanized. The entire stomach was carefully removed and then opened on its lesser curvature longitudinally to expose the gastric mucosa. The mucosal layers were harvested with a glass slide. The scraping samples were stored at $-80^{\circ} \mathrm{C}$ before the analysis. To prepare paraffin sections, the stomach from each group was carefully isolated and immediately fixed in 10\% neutral buffered formalin before embedding. Gastric mucosal frozen specimens of matched normal volunteer and PHG patients with cirrhosis were obtained from the endoscopic unit of The Third Affiliated Hospital of Sun Yat-Sen University. The acquisition of the tissue samples was approved by the Institutional Review Board at The Third Affiliated Hospital of Sun Yat-Sen University. Written informed consent was received from each patient before inclusion in the study.

Cell culture, drug administration and siRNA treatment. The GES-1 and SGC7901 cell lines were obtained from the American Type Culture Collection (ATCC, Manassas, VA, USA). The cells were cultured in RPMI medium 1640 supplemented with $10 \%$ heat-inactivated fetal bovine serum, 100 units $/ \mathrm{ml}$ penicillin and $100 \mu \mathrm{g} / \mathrm{ml}$ streptomycin in a humidified incubator at $37^{\circ} \mathrm{C}$ with $5 \%$ $\mathrm{CO}_{2}$. Tunicamycin ( $100 \mathrm{ng} / \mathrm{ml}$, Sigma, St. Louis, MO, USA) was added after these cells had grown to a density of $90 \%$ to induce ER stress. For siRNA treatment, the cells were transfected with $20 \mu \mathrm{M}$ PUMA RNA oligo kit (GenePharma, Shanghai, China). Two PUMA-siRNA sequences were purchased and according to the manufacturer's instructions, the most effective sequence was selected to be able to achieve a transfection efficiency of $>90 \%$. After incubation for $24 \mathrm{~h}$, the transfection medium was replaced by regular culture medium before tunicamycin administration.

Histological and TUNEL staining. For histological and TUNEL analysis, formalin fixed tissues were embedded in paraffin and sectioned. The $4 \mathrm{~mm}$ sections were stained by hematoxylin and eosin (H\&E). TUNEL staining was performed using an in situ cell death detection kit (Roche, Basel, Switzerland) according to the manufacturer's instructions. The apoptotic index was determined by dividing the number of apoptotic cells by the total number of cells in the mucosa of at least 20 randomly selected fields $(\times 200)$. Three mice or human tissues from each group were studied.

IHC staining, IF staining and Van Gieson staining. The frozen tissues from three randomly selected animals from each group were used to prepare the sections. For IHC staining, $4 \mu \mathrm{m}$ frozen sections were fixed with $4 \%$ paraformaldehyde in phosphate-buffered saline (PBS) for $15 \mathrm{~min}$, then permeabilized with $1 \%$ Triton X-100 for 30 min and washed three times with PBS. Next, the targeted protein was detected using secondary antibodies followed by detection using the ABC staining system (Santa Cruz, Santa Cruz, CA, USA), and the sections were counterstained with hematoxylin. For IF staining, the targeted protein was detected by related secondary antibody. Antibody-antigen complexes were visualized by incubation with biotin-conjugated secondary antibody and streptavidin Alexa 488 or 594 (Molecular Probes, Eugene, OR, USA), with the nuclei counterstained with $2 \mathrm{mg} / \mathrm{ml}$ of $4^{\prime} 6$-diamidino-2-phenylindole (DAPI) dihydrochloride (Molecular Probes). For double staining, after finishing the first protein detection, the slides were used to detect the secondary protein. $\mathrm{IHC}$, IF and double staining was performed by using antibodies for PUMA (Abcam, Cambridge, MA, USA), GRP78 (Enzo Life Sciences, Lausen, Switzerland), cytokeratin (Abcam) and cleaved caspase-3 (Cell Signaling Technology, Danvers, MA, USA). Fiber and collagen in tissue sections from liver cirrhosis were detected using the Van Gieson staining kit (Baso, Zhuhai, China) according to the manufacturer's instructions.

Analysis of Bax/Bak translocation. To analyze Bax/Bak translocation, an aliquot of each gastric mucosal scraping sample was used to isolate mitochondrial and cytosolic fractions by the differential centrifugation method as previously described. ${ }^{29}$

Western blotting. Total protein extraction and mitochondrial and cytosolic fractions were analyzed by western blotting. Antibodies used for western blotting included antibodies that recognize PUMA, GRP78, sXBP-1 (Novus Biologicals, Littleton, CO, USA), elF2 $\alpha$, p-elF2 $\alpha$, cleaved caspase-12, cleaved caspase-4, cleaved caspase-9, cleaved caspase-3, Cox IV (all from Cell Signaling Technology), cytochrome $c$ (Santa Cruz), Bax, Bak (Abcam) and $\beta$-actin (Sigma). Appropriate horseradish peroxidase-conjugated secondary antibodies were used to detect the primary antibody/antigen complexes. The signal was detected using ECL Western blotting detection reagents (Amersham Pharmacia Biotech, Piscataway, NJ, USA). After quantifying the signals by densitometry, the results are expressed as a ratio to loading control densitometry units.

Total RNA extraction and real-time PCR. Total RNA was isolated from the gastric mucosal scraping samples using the RNAgents Total RNA Isolation System (Promega, Madison, WI, USA) according to the manufacturer's instructions. First-strand CDNA was synthesized using Superscript Reverse Transcriptase (Invitrogen, Carlsbad, CA, USA) according to the manufacturer's instructions. Real-time PCR was performed on a Chromo 4 Detector System (MJ Research, Sierra Point, CA, USA) using gene-specific primers and DyNAmo SYBR Green Master Mix (Finnzymes, Espoo, Finland). PUMA was amplified using primers PUMA-exon 3F, $5^{\prime}$-ATGGCGGACGACCTCAAC- $3^{\prime}$ and PUMA-exon 4R, $5^{\prime}$-AGTCCCATGAAGAGATTGTACATGAC-3' to yield the 103-bp product. As the internal control, the expression of $\beta$-actin in each sample was also quantified using the sense primers $5^{\prime}$-GTGGGCCGCTCTAGGCACCA-3' and the antisense primer $5^{\prime}$-CGGTTGGCCTTAGGGTTCAGGGGGG-3' (242 bp product). Three mice or human tissues were used in each group. PCR products were analyzed by agarose gel electrophoresis.

Statistical analysis. The data are expressed as the means \pm standard deviation and were evaluated by one-way ANOVAs in which multiple comparisons were performed using the method of least significant difference. Differences were considered as statistically significant if the probability of the difference occurring by chance was $<5$ in $100(P<0.05)$.

\section{Conflict of Interest}

The authors declare no conflict of interest.

Acknowledgements. We thank Professor Chunkui Shao (Division of Pathology, The Third Affiliated Hospital of Sun Yat-Sen University) for excellent technical assistance and helpful discussion. Supported partly by the Major Projects Incubator Program of National Key Basic Research Program of China (2012CB526700), the National Natural Science Foundation of China (30971357, 81370511), the Natural Science Foundation of Guangdong Province (S2011020002348), the Science and Technology Planning Project of Guangdong Province (2009B060300001), the International Cooperative Innovative Platform of Guangdong Province Universities and Colleges (gihz1101), the Projects of Guangzhou City International Cooperation (2012J5100017), the Fundamental Research Funds for the Central Universities (13ykjc01), and 985 Program (82000-3281901). 
1. McCormack TT, Sims J, Eyre-Brook I, Kennedy H, Goepel J, Johnson AG et al. Gastric lesions in portal hypertension: inflammatory gastritis or congestive gastropathy? Gut 1985; 26: 1226-1232.

2. Cubillas R, Rockey DC. Portal hypertensive gastropathy: a review. Liver Int 2010; 30: 1094-1102.

3. Shertsinger AG, Zhigalova SB, Semenova TS, Tsaava DV. [Portal gastropathy: diagnosis, classification]. Eksp Klin Gastroenterol 2012; 6: 62-68.

4. Kawanaka H, Tomikawa M, Jones MK, Pai R, Szabo IL, Sugimachi K et al. Portal hypertensive gastric mucosa has reduced activation of MAP kinase (ERK2) in response to alcohol injury: a key to impaired healing? FASEB J 2001; 15: 574-576.

5. Payen JL, Cales P, Pienkowski P, Sozzani P, Kervran A, Frexinos $J$ et al. Weakness of mucosal barrier in portal hypertensive gastropathy of alcoholic cirrhosis. Effects of propranolol and enprostil. J Hepatol 1995; 23: 689-696.

6. Muñoz J, Albillos A, Pérez-Páramo M, Rossi I, Alvarez-Mon M. Factors mediating the hemodynamic effects of tumor necrosis factor- $\alpha$ in portal hypertensive rats. Am J Physiol 1999; 276: G687-G693.

7. Wu B, Zeng L, Lin Y, Wen Z, Chen G, Iwakiri R et al. Downregulation of cyclooxygenase-1 is involved in gastric mucosal apoptosis via death signaling in portal hypertensive rats. Cell Res 2009; 19: 1269-1278.

8. Wang JY, Hsieh JS, Huang TJ. The effect of portal hypertension on transforming growth factor- $\alpha$ and epidermal growth factor receptor in the gastric mucosa of rats. Int Surg 1998; 83: 220-223.

9. Ouyang L, Shi Z, Zhao S, Wang FT, Zhou TT, Liu B et al. Programmed cell death pathways in cancer: a review of apoptosis, autophagy and programmed necrosis. Cell Prolif 2012; 45: 487-498.

10. Gupta S. Molecular signaling in death receptor and mitochondrial pathways of apoptosis. Int J Oncol 2003; 22: 15-20.

11. Chipuk JE, Green DR. PUMA cooperates with direct activator proteins to promote mitochondrial outer membrane permeabilization and apoptosis. Cell Cycle 2009; 8: 2692-2696.

12. Thakur VS, Ruhul Amin AR, Paul RK, Gupta K, Hastak K, Agarwal MK et al. p53-Dependent p21-mediated growth arrest pre-empts and protects HCT116 cells from PUMA-mediated apoptosis induced by EGCG. Cancer Lett 2010; 296: 225-232.

13. Shaltouki A, Freer M, Mei $Y$, Weyman $C M$. Increased expression of the pro-apoptotic $\mathrm{Bcl} 2$ family member PUMA is required for mitochondrial release of cytochrome $\mathrm{C}$ and the apoptosis associated with skeletal myoblast differentiation. Apoptosis 2007; 12: 2143-2154.

14. Yu J, Zhang L. PUMA, a potent killer with or without p53. Oncogene 2008; 27(Suppl 1): S71-S83.

15. Zou CG, Cao XZ, Zhao YS, Gao SY, Li SD, Liu XY et al. The molecular mechanism of endoplasmic reticulum stress-induced apoptosis in $\mathrm{PC}-12$ neuronal cells: the protective effect of insulin-like growth factor I. Endocrinology 2009; 150: 277-285.

16. Reimertz C, Kögel D, Rami A, Chittenden T, Prehn JH. Gene expression during ER stressinduced apoptosis in neurons: induction of the BH3-only protein Bbc3/PUMA and activation of the mitochondrial apoptosis pathway. J Cell Biol 2003; 162: 587-597.

17. Klomporn P, Panyasrivanit M, Wikan N, Smith DR. Dengue infection of monocytic cells activates ER stress pathways, but apoptosis is induced through both extrinsic and intrinsic pathways. Virology 2011; 409: 189-197.

18. Szegezdi E, Duffy A, O'Mahoney ME, Logue SE, Mylotte LA, O'brien T et al. ER stress contributes to ischemia-induced cardiomyocyte apoptosis. Biochem Biophys Res Commun 2006; 349: 1406-1411.

19. McGuckin MA, Eri RD, Das I, Lourie R, Florin TH. ER stress and the unfolded protein response in intestinal inflammation. Am J Physiol Gastrointest Liver Physiol 2010; 298: G820-G832.
20. Gardner BM, Pincus D, Gotthardt K, Gallagher CM, Walter P. Endoplasmic reticulum stress sensing in the unfolded protein response. Cold Spring Harb Perspect Biol 2013; 5: a013169.

21. Gorman AM, Healy SJ, Jäger R, Samali A. Stress management at the ER: regulators of ER stress-induced apoptosis. Pharmacol Ther 2012; 134: 306-316.

22. Yu J, Wang P, Ming L, Wood MA, Zhang L. SMAC/Diablo mediates the proapoptotic function of PUMA by regulating PUMA-induced mitochondrial events. Oncogene 2007; 26: 4189-4198.

23. Kamath PS, Lacerda M, Ahlquist DA, McKusick MA, Andrews JC, Nagorney DA. Gastric mucosal responses to intrahepatic portosystemic shunting in patients with cirrhosis. Gastroenterology 2000; 118: 905-911.

24. Ohta M, Yamaguchi S, Gotoh N, Tomikawa M. Pathogenesis of portal hypertensive gastropathy: a clinical and experimental review. Surgery 2002; 131: S165-S170.

25. Watanabe D, Otaka M, Mikami K, Yoneyama K, Goto T, Miura K et al. Expression of $72-\mathrm{kDa}$ heat shock protein, and its cytoprotective function, in gastric mucosa in cirrhotic rats. J Gastroenterol 2004; 39: 724-733.

26. Chen D, Zheng X, Kang D, Yan B, Liu X, Gao Y et al. Apoptosis and expression of the Bcl-2 family of proteins and P53 in human pancreatic ductal adenocarcinoma. Med Princ Pract 2012; 21: 68-73.

27. Hardwick JM, Soane L. Multiple functions of BCL-2 family proteins. Cold Spring Harb Perspect Biol 2013; 5: a008722.

28. Han J, Flemington C, Houghton AB, Gu Z, Zambetti GP, Lutz RJ et al. Expression of bbc3, a pro-apoptotic $\mathrm{BH} 3-$ only gene, is regulated by diverse cell death and survival signals. Proc Natl Acad Sci USA 2001; 98: 11318-11323.

29. Wu B, Qiu W, Wang P, Yu H, Cheng T, Zambetti GP et al. p53 independent induction of PUMA mediates intestinal apoptosis in response to ischaemia-reperfusion. Gut 2007; 56 : 645-654.

30. Spender LC, Carter MJ, O'Brien DI, Clark LJ, Yu J, Michalak EM et al. Transforming growth factor- $\beta$ directly induces p53-up-regulated modulator of apoptosis (PUMA) during the rapid induction of apoptosis in myc-driven B-cell lymphomas. J Biol Chem 2013; 288: 5198-5209.

31. Chipuk JE, Fisher JC, Dillon CP, Kriwacki RW, Kuwana T, Green DR. Mechanism of apoptosis induction by inhibition of the anti-apoptotic BCL-2 proteins. Proc Natl Acad Sci USA 2008; 105: 20327-20332.

32. Wallace JL. Prostaglandins, NSAIDs, and gastric mucosal protection: why doesn't the stomach digest itself? Physiol Rev 2008; 88: 1547-1565.

33. Ghosh AP, Klocke BJ, Ballestas ME, Roth KA. CHOP potentially cooperates with FOXO3a in neuronal cells to regulate PUMA and BIM expression in response to ER stress. PLoS One 2012; 7: e39586.

34. Nickson P, Toth A, Erhardt P. PUMA is critical for neonatal cardiomyocyte apoptosis induced by endoplasmic reticulum stress. Cardiovasc Res 2007; 73: 48-56.

35. Kieran D, Woods I, Villunger A, Strasser A, Prehn JH. Deletion of the BH3-only protein puma protects motoneurons from ER stress-induced apoptosis and delays motoneuron loss in ALS mice. Proc Natl Acad Sci USA 2007; 104: 20606-20611.

36. Futami T, Miyagishi M, Taira K. Identification of a network involved in thapsigargin-induced apoptosis using a library of small interfering RNA expression vectors. J Biol Chem 2005; 280: $826-831$.

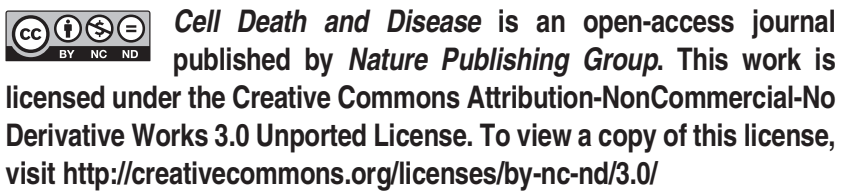

